\title{
Analysis of Autophagy-Related Signatures in The Tumor Immune Microenvironment and Identification of Clinical Prognostic Markers in Bladder Carcinoma
}

\section{Huifeng Cao}

First Affiliated Hospital of Jiamusi University

\section{Dayin Chen}

First Affiliated Hospital of Jiamusi University

Zhihui Zhang

Hongqi Hospital Affiliated to Mudanjiang Medical College

Liang Cheng

First Affiliated Hospital of Jiamusi University

Zhenguo Luo

First Affiliated Hospital of Jiamusi University

Junjuan Yu ( a1169207591@163.com )

First Affiliated Hospital of Jiamusi University

\section{Research Article}

Keywords: bladder carcinoma, prognosis prediction model, autophagy, tumor microenvironment

Posted Date: December 29th, 2021

DOI: https://doi.org/10.21203/rs.3.rs-1187613/v1

License: (c) (i) This work is licensed under a Creative Commons Attribution 4.0 International License.

Read Full License 


\section{Abstract}

Objectives: Bladder carcinoma (BLCA) is one of the most common malignant diseases of urinary system. Our study aimed to investigate the autophagy-related signatures in the tumor immune microenvironment and construct effective prognosis prediction model.

Methods: RNA sequencing data and corresponding clinical information of BLCA were downloaded from The Cancer Genome Atlas (TCGA) and Gene Expression Omnibus (GEO). Autophagy-related genes were extracted from TCGA dataset for consensus clustering analysis, and differences in survival rate were analyzed. STIMATE algorithm was used to analyze the tumor microenvironment (TME) and immune cell infiltration was compared between different clusters. Differentially expressed genes (DEGs) between different clusters were identified, followed by function annotation. Independent prognostic signatures were further revealed to construct prognostic prediction model.

Results: We identified 35 autophagy-related genes associated with prognosis. Survival rate of samples in cluster 1 was significant lower than that in cluster 2. Cluster 2 had markedly lower tumor purity and significantly higher estimate score and stromal score than cluster 1 . The proportions of T cells CD8, macrophages M1, T cells CD4 memory activated, NK cells activated, and dendritic cells activated were higher in cluster 1 . There were 1,275 DEGs which were mainly enriched in functions and pathways related to immune response and cancer. Seven genes (ATF6, CAPN2, NAMPT, NPC1, P4HB, PIK3C3, and RPTOR) were further identified as the independent prognostic signatures to construct risk score prediction model, which had good prediction performance.

Conclusion: Prognosis prediction model based on 7 autophagy-related genes may have great value in BLCA prognosis prediction.

\section{Introduction}

Bladder carcinoma (BLCA) is one of the most common malignant diseases of urinary system and its incidence is increasing worldwide, with an estimated of 573,000 new cases diagnosed and approximately 213,000 deaths caused in 2020 [1, 2]. Among the diagnosed BLCA patients, 70\% of patients present with superficial tumors at the time of diagnosis and $30 \%$ of patients present as muscle-invasive disease associated with a high risk of death [3]. Despite great progress has been made in cancer biology and treatment for BLCA, such as surgery, immunotherapy, radiotherapy, and chemotherapy, the survival outcomes of the patients diagnosed with muscle-invasive and advanced BLCA remain poor $[4,5]$. Therefore, it is urgently to find the prognostic markers to guide patient treatment selection and construct the effective prognosis prediction model for BLCA.

The tumor microenvironment (TME) has great importance in the tumor progression and the drug resistance of BLCA due to the heterogeneity and complexity of tumor-associated stromal cells and the extracellular milieu [6]. It has been shown that stratification of TME immune types is beneficial for selection of immunotherapeutic strategies in tumor [7]. What's more, novel insights into the molecular 
mechanisms of immune evasion in BLCA are essential for developing immunotherapy that is targeting specific components of TME [8]. According to an analysis of 542 patients with muscle-invasive bladder cancer, quantities and spatial organization of stromal tumor-infiltrating lymphocytes within TME could predict stages of tumor inflammation and patient survival [9].

Autophagy is an evolutionary catabolic mechanism to maintain cellular environmental homeostasis by recycling and degrading internal constituents, including macromolecules and organelles [10]. Recent findings have revealed that autophagy plays an important role in the malignant transformation and is proposed as a significant factor in tumor development $[11,12]$. Furthermore, autophagy may affect TME by preventing cytotoxicity and providing sustainable source of biomolecules and cellular energy demands under stressful conditions [13, 14]. In the study of Li et al., immune-related prognostic genes for BLCA were identified by analyzing the relationship between TME and gene expression profiles of one dataset from Gene Expression Omnibus (GEO), while the roles of autophagy in TME was not explored based on gene expression profiles [15].

In order to further reveal autophagy-related signatures in TME and uncover the potential molecular mechanisms, our current study included 394 BLCA samples as training dataset from The Cancer Genome Atlas (TCGA), and four datasets from GEO as validation dataset. Autophagy-related genes were firstly revealed and samples were clustered based on autophagy-related signatures, followed by differential expression and tumor immune microenvironment analysis. Moreover, prognostic prediction model was constructed based on autophagy-related signatures and compared with clinical prognostic prediction model (Figure 1).

\section{Materials And Methods}

\section{Data source and preprocessing}

RNA sequencing data and corresponding clinical information of BLCA were downloaded from TCGA (https://portal.gdc.cancer.gov/) and GEO (https://www.ncbi.nlm.nih.gov/geo/). The downloaded dataset from TCGA was consisted of 430 samples, including 411 tumor samples and 19 normal control samples. After the samples without overall survival (OS) value or OS $<30$ days were removed, we obtained 394 samples which were considered as training dataset. Meanwhile, there were four available datasets (GSE13507, GSE48075, GSE48276 and GSE69795) of BLCA from GEO and considered as validation dataset. Data normalization was conducted by normalizeBetweenArrays function in Limma (Linear Models for Microarray Data) package (https://bioconductor.org/packages/release/bioc/html/limma.html) [16] of R. The clinical information of TCGA and GEO datasets were listed in supplementary table 1.

\section{Consensus clustering analysis of autophagy-related genes}

There were 232 autophagy-related genes in human autophagy database (HADb, http://autophagy.lu/clustering/index.html; supplementary table 2) [17]. A total of 214 autophagy-related 
genes with non-zero expressions were extracted from TCGA dataset. Afterwards, autophagy-related genes with median absolute deviation $(M A D)<0.05$ were excluded. The remained autophagy-related genes were submitted to univariate Cox regression analysis by Survival package (http://bioconductor.org/packages/survivalr/) [18]. Clustering analysis was performed for autophagyrelated genes with MAD $>0.05$ and log-rank $p$ value $<0.05$ by ConsensuClusterPlus [19] in $R$ based on Euclidean and Ward's linkage, with cycle computation 1,000 times to ensure stability and reliability. Samples were classified into two different clusters by optimal k-means clustering and further confirmed using t-distributed stochastic neighbour embedding (t-SNE) algorithm [20]. The differences in survival rate between different clusters were analyzed using the Kaplan-Meier method [21].

\section{Differentially expressed genes and function annotation}

DEGs between the two clusters were identified by Limma package [17], with the cut-off criteria of $\|$ og2 fold change (FC) $\mid>0.5$ and adjusted $p<0.05$. Gene ontology (GO) functional enrichment in terms of biological process (BP), cellular component (CC), and molecular function (MF) as well as Kyoto Encyclopedia of Genes and Genomes (KEGG) pathway enrichment analysis were conducted for differentially expressed genes via the Database for Annotation, Visualization and Integrated Discovery (DAVID, https://david.ncifcrf.gov/) [22] with a threshold of $p<0.05$.

\section{Immune cell infiltration comparison between two clusters}

We applied the ESTIMATE algorithm [23] and downloaded R script (https://sourceforge.net/projects/estimateproject/) to calculate the estimate scores, stromal scores, and immune scores, which were used to predict tumor purity and analyze the TME. In order to compare the differences in immune cell subtypes between the two clusters, the CIBERSORT package was used to calculate the proportions of 22 immune cell subtypes for TCGA samples. Samples with $p<0.05$ in CIBERSORT analysis were used for further analysis, and the Mann-Whitney $U$ test was used to compare the differences between the two clusters.

\section{Construction of prognostic prediction model based on autophagy-related genes}

Autophagy-related genes related to BLCA risk was identified by Lasso Cox regression analysis via the glmnet software package of $R$, and independent prognostic signatures were further revealed by multivariable Cox regression. The risk score was calculated based on the identified prognostic signature genes according to the following formula:

Risk score $(R S)=\sum \beta_{i} \times \operatorname{Exp}_{i}$

Where $\beta_{\mathrm{i}}$ referred to the multivariable Cox regression coefficients of signatures, and $\operatorname{Exp}_{\mathrm{i}}$ denoted the expression levels of signatures.

The receiver operating characteristic (ROC) curves were plotted by survivalROC package in $\mathrm{R}$, and area under the curve (AUC; $A U C=0.5$ : no discriminatory power, $A U C=1$ : perfect discriminatory power) was 
used to evaluate the prognostic accuracy of the prediction model.

\section{Statistical analysis}

R software (Version 4.1.0; https://bioconductor.org/packages/release/bioc/html/limma.html) was used for statistical analysis. The differences in OS between groups were analyzed by Kaplan-Meier curves and log-rank test. Lasso Cox regression was applied to reveal the correlations of autophagy-related genes with prognosis, and multivariable Cox regression was used to identify the independent prognostic signatures. The performance of prediction model was evaluated by ROC curves and AUC. Mann-Whitney $\mathrm{U}$ test was used to compare the differences between the two clusters. All statistical p-values were bilateral, and $p<0.05$ was considered statistically significant.

\section{Results}

\section{Consensus clustering of autophagy-related genes in two clusters with different clinical outcomes of BLCA}

Among the 232 autophagy-related genes in HADb, 214 genes with MAD > 0.5 extracted from TCGA dataset were used to perform univariate Cox regression analysis and 35 candidate genes associated with prognosis with $\mathrm{p}<0.05$ were obtained, such as APOL 1, CTSB, P4HB, ITGB1, and EIF4G1 (Table 1). By using the ConsensusClusterPlus in R, the TCGA samples were clustered into different groups based on the expression levels of these 35 autophagy-related genes. The crossover between the BLCA samples was the least when the consensus matrix k value was 2 (two subclasses: cluster 1 and cluster 2), the consensus matrix heat map showed a clear boundary (Figure 2A), and the crossover between the BLCA samples was the least (Figure 2B, 2C). The samples clustered into cluster 1 or cluster 2 were listed in supplementary table 3 .

Meanwhile, t-SNE algorithm was used to reduce the dimensionality of features, and the two subclasses were largely consistent with the two dimensional pattern of t-SNE distribution (Figure 2D). Heatmap of two clusters defined by the autophagy-related genes showed that the expressions between the two clusters were different (Figure 2E). Kaplan-Meier curves showed that the survival rate of samples in cluster 1 was significant lower than that in cluster 2 with a log-rank $p<0.01$ (Figure 2F).

\section{Enriched functions related to immune and tumor for DEGs between two clusters}

By using the Limma package, 1,275 DEGs were identified, including 637 up-regulated genes and 638 down-regulated genes in cluster 1 by comparing with the cluster 2 (supplementary table 4). Functional enrichment analysis showed that DEGs were enriched in the GO terms related to immune response and cancer. DEGs were mainly related to immune responses of viral process, positive regulation of I-kappaB kinase/N-kappaB signaling, and interferon-gamma-mediated signaling pathway (Figure 3A) (supplementary table 5). The enriched GO terms related to cancer were apoptotic process, cell proliferation, and angiogenesis (Figure 3B) (supplementary table 5). The DEGs were significantly involved in pathways related to tumor such as pathways in cancer, VEGF signaling pathway, cell cycle, 
proteoglycans in cancer, HTLV-I infection, MAPK signaling pathway, and PI3K-Akt signaling pathway (Supplementary figure 1) (supplementary table 5).

\section{Immune characteristics of the two clusters}

After the estimate scores, immune scores, stromal scores, and tumor purity of the two clusters were achieved, we found the estimate score $(p=0.0325)$ and stromal score $(p=1.46 e-04)$ of cluster 2 were obviously higher than those of the cluster 1 , while significantly lower tumor purity $(p=0.0325)$ was observed in cluster 2 (Figure 4A).

The proportions of 22 immune cell subtypes were calculated by CIBERSORT package, and cluster 2 had significantly higher proportions of the macrophages $\mathrm{M} 2$, macrophages $\mathrm{M} 0$, $\mathrm{T}$ cells CD4 memory resting, $\mathrm{T}$ cells regulatory (Tregs), and B cells naive (Figure 4B). The proportions of T cells CD8, macrophages M1, $T$ cells CD 4 memory activated, NK cells activated, and dendritic cells activated were lower in cluster 2 by comparing with the cluster 1 (Figure 4B) .

\section{Prognostic model based on autophagy-related genes had good prediction performance}

Lasso Cox regression analysis was performed for the 35 autophagy-related genes to minimize the risk of overfitting and 24 genes were obtained (Figure 5A, B). The regression coefficients of these 24 genes were listed in supplementary table 6. Multivariable Cox regression indicated 7 genes (ATF6, CAPN2, NAMPT, NPC1, P4HB, PIK3C3, and RPTOR) were the independent prognostic signatures (Table 2; Figure $5 \mathrm{C}$ ). The coefficients and expressions of these 7 genes were used to calculate the risk score. Samples were separated into low-risk or high-risk groups with the median risk score as cutoff value. Kaplan-Meier curves showed that high-risk group had poorer prognosis $(p=0.00019$; Figure 5D). With the increasing of risk score, the number of dead patients elevated (Figure 5E). According to the univariate and multivariate Cox regression analysis, age, stage and risk score were found to be independent prognostic factors (Figure 5F). ROC curves of risk score, age, gender, stage and grade indicated that risk score had the best prediction performance in 1 year survival probability $(A U C=0.671)$ (Figure 5G).

\section{Autophagy-related genes in GEO dataset}

Autophagy-related genes were extracted from GSE13507, GSE48075, GSE48276 and GSE69795. Univariate Cox regression analysis was used to reveal the autophagy-related genes associated with prognosis, and MTOR was the overlapping gene with the 35 autophagy-related genes in TCGA dataset (Supplementary figure 2). The ROC curves showed that MTOR expression levels were significantly related to tumor risk with $p<0.05$ in GEO datasets (Figure 6).

\section{Discussion}

BLCA is one of the top ten most common forms of cancer in the world, and the treatment burden of BLCA is high due to drug resistance, frequent surveillance strategies, high recurrence and metastasis rate [24, 
25]. Cancer stem cells factors, genetic heterogeneity, autophagy, and TME are involved in the drug resistance of cancer cells, and autophagy may affect the regulation of cancer stem cell homeostasis and TME, [26, 27]. Therefore, it is of vital importance to understand the potential mechanism of autophagy and TME regulation in the BLCA.

In our study, 35 autophagy-related genes associated with prognosis were revealed for sample clustering, and the survival rate of samples in cluster 1 was significant lower than that in cluster 2 based on the Kaplan-Meier curves. According to ESTIMATE algorithm, the estimate score and stromal score were significantly higher in cluster 2 and tumor purity were markedly lower. In the study of Li et al, they found stromal scores were associated with clinical characteristics and prognosis of BLCA patients [15]. This result of our study is consistent with previous study, which indicated that the TME composition may influence the prognosis of BLCA patients.

Previous researchers have indicated that macrophages in tumor tissues play an indispensable role in tumor immunity and progression depending on the cytokine microenvironment [28]. However, the specific molecular mechanisms of tumor-associated macrophages in the modulation of TME remain poorly understood. In our current study, comparison of the immune cell proportions in the TME between the two clusters showed that the cluster 2 had significantly higher proportions of the macrophages M2, macrophages M0, T cells CD4 memory resting, T cells regulatory (Tregs), and B cells naïve. Meanwhile, the proportions of T cells CD8, macrophages M1, T cells CD 4 memory activated, NK cells activated, and dendritic cells activated were lower in cluster 2 . These results suggested that the immune cell infiltration was activated in patients with poor prognosis.

A total of 1,275 DEGs were identified between cluster 1 and cluster 2 . Functional enrichment analysis showed that DEGs were enriched in the GO terms related to immune response and cancer, such as positive regulation of I-kappaB kinase/N-kappaB signaling, interferon-gamma-mediated signaling pathway, apoptotic process, cell proliferation, and angiogenesis. Bacterial immunotherapy for BLCA has the ability to induce tumor immunity causing anti-tumor effects that result from enhanced CD4 T cells, and ultimately requires tumor-intrinsic interferon-gamma signaling [29]. Moreover, DEGs were significantly involved in pathways related to tumor such as pathways in cancer, VEGF signaling pathway, cell cycle, proteoglycans in cancer, HTLV-I infection, MAPK signaling pathway, and PI3K-Akt signaling pathway. These results indicated that the DEGs between cluster 1 and cluster 2 classified by 35 autophagy-related genes participate in BLCA by affecting the immune microenvironment.

By using Lasso Cox regression and multivariable Cox regression analysis, 7 genes (ATF6, CAPN2, NAMPT, NPC1, P4HB, PIK3C3, and RPTOR) among the 35 autophagy-related genes were identified as the independent prognostic signatures to establish risk score prediction model. Kaplan-Meier curves showed that high-risk group had poorer prognosis. Activating transcription factor 6 (ATF6) is a member of the leucine zipper family and regulates endoplasmic reticulum stress [30]. It has shown that high expression of otubain 1 (OTUB1) promotes BLCA progression by stabilizing ATF6 under endoplasmic reticulum stress [31]. Cancer cell chemoresistance is associated with the ATF6-induced autophagy, resulting poor 
overall survival [32]. Nicotinamide phosphoribosyl transferase (NAMPT) is the rate limiting enzyme in procancer NAD+ synthesis, and it showed that inhibition of nicotinamide phosphoribosyl transferase (NAMPT) can suppress the growth of differentiated cancer cells through autophagy [33]. Genetic variants in NAMPT have been confirmed associated with BLCA risk and could be considered as genetic biomarker for the BLCA prognosis [34]. Previous study demonstrated that MPTOL145 which is a first-in-class PIK3C3/FGFR inhibitor has potent anti-bladder cancer activity, which enhances autophagosome formation by FGFR inhibition and impairs autophagy flux via PIK3C3 inhibition [35]. What's more, blocking pro-survival autophagy by targeting PIK3C3 with MPTOL145 can significantly improve the sensitivity of cancer cells to targeted drugs or chemotherapeutic drugs, and promote the rational combination strategy of cancer treatment [36]. These results indicated that these 7 genes might be the potential targets in developing novel therapies for BLCA.

Univariate and multivariate Cox regression analysis indicated that age, stage, and risk score were independent prognostic factors. ROC curves of risk score, age, gender, stage and grade in 1, 3,5 years survival probability indicated that risk score had the best prediction performance in 1 year survival probability and had good prediction performance in 3 , and 5 years survival probability. Among the 35 autophagy-related genes related to prognosis in TCGA dataset, MTOR was the overlapping gene between GEO datasets and TCGA dataset. The ROC curves showed that MTOR expression levels were significantly related to tumor risk in GEO datasets.

In conclusion, 35 autophagy-related genes associated with prognosis were revealed and 1,275 DEGs were identified between cluster 1 and cluster 2 classified by 35 autophagy-related genes. Cluster 2 had markedly lower tumor purity and significantly higher estimate score and stromal score than cluster 1 . The proportions of T cells CD8, macrophages M1, T cells CD 4 memory activated, NK cells activated, and dendritic cells activated were higher in cluster 1 . The identified DEGs were mainly enriched in functions and pathways related to immune response and cancer. Moreover, 7 genes (ATF6, CAPN2, NAMPT, NPC1, $P 4 H B, P I K 3 C 3$, and RPTOR) were further identified as the independent prognostic signatures and risk score prediction model was constructed, which had good prediction performance.

\section{Declarations}

Ethics approval and consent to participate: Not applicable.

Consent for publication: Not applicable.

Acknowledgements: Not applicable.

Availability of data and materials: The datasets generated and/or analysed during the current study are available in the TCGA repository, https://portal.gdc.cancer.gov/ and GEO repository, https://www.ncbi.nlm.nih.gov/geo/

Competing interests: The authors declare that they have no competing interests. 
Funding: Not applicable.

Authors' contributions: Huifeng Cao and Junjuan Yu contributed to the conception and design of the study. Huifeng Cao performed the data acquisition. Dayin Chen and Zhihui Zhang performed the statistical analysis. Liang Cheng and Zhenguo Luo analyzed and interpreted the data. Huifeng Cao drafted the manuscript. Junjuan Yu revised the manuscript. All authors read and approved the final manuscript.

\section{References}

1. Siegel RL, Miller KD, Jemal A. Cancer statistics, 2020. CA-Cancer J Clin 2020;70, (1), 7-30.

2. Sung H, Ferlay J, Siegel RL, Laversanne M, Soerjomataram I, Jemal A, et al. Global Cancer Statistics 2020: GLOBOCAN Estimates of Incidence and Mortality Worldwide for 36 Cancers in 185 Countries. CACancer J Clin 2021;71, (3), 209-249.

3. Kaufman DS, Shipley WU, Feldman AS. Bladder cancer. Lancet 2009, 374, (9685), 239-49.

4. Patel VG, Oh WK, Galsky MD. Treatment of muscle-invasive and advanced bladder cancer in 2020. CACancer J Clin 2020, 70, (5), 404-423.

5. Rouanne M, Bajorin DF, Hannan R, Galsky MD, Williams SB, Necchi A, et al. Rationale and Outcomes for Neoadjuvant Immunotherapy in Urothelial Carcinoma of the Bladder. Eur Urol Oncol 2020, 3, (6), 728-738.

6. Lee HW, Chung W, Lee H-O, Jeong DE, Jo A, Lim JE, et al. Single-cell RNA sequencing reveals the tumor microenvironment and facilitates strategic choices to circumvent treatment failure in a chemorefractory bladder cancer patient. Genome Med 2020, 12, (1), 47-47.

7. Chen YP, Zhang Y, Lv JW, Li YQ, Wang YQ, He QM, et al. Genomic Analysis of Tumor Microenvironment Immune Types across 14 Solid Cancer Types: Immunotherapeutic Implications. Theranostics 2017, 7, (14), 3585-3594.

8. Crispen PL, Kusmartsev S. Mechanisms of immune evasion in bladder cancer. Cancer Immunol Immunother 2020, 69, (1), 3-14.

9. Pfannstiel C, Strissel PL, Chiappinelli KB, Sikic D, Wach S, Wirtz RM,et al. The Tumor Immune Microenvironment Drives a Prognostic Relevance That Correlates with Bladder Cancer Subtypes. Cancer Immunol Res 2019, 7, (6), 923-938.

10. Galluzzi L, Green DR. Autophagy-Independent Functions of the Autophagy Machinery. Cell 2019, 177, (7), 1682-1699.

11. Poillet-Perez L, White E. Role of tumor and host autophagy in cancer metabolism. Genes Dev 2019, 33, (11-12), 610-619. 
12. Li X, He S, Ma B. Autophagy and autophagy-related proteins in cancer. Mol Cancer 2020, 19, (1), $020-$ 1138.

13. Kocaturk NM, Akkoc Y, Kig C, Bayraktar O, Gozuacik D, Kutlu O. Autophagy as a molecular target for cancer treatment. Eur J Pharm Sci 2019, 134, 116-137.

14. Li F, Guo H, Yang Y, Feng M, Liu B, Ren X, et al. Autophagy modulation in bladder cancer development and treatment (Review). Oncol Rep 2019, 42, (5), 1647-1655.

15. Li F, Teng H, Liu M, Liu B, Zhang D, Xu Z, et al. Prognostic Value of Immune-Related Genes in the Tumor Microenvironment of Bladder Cancer. Front Oncol 2020, 10, 1302-1302.

16. Ritchie ME, Phipson B, Wu D, Hu Y, Law CW, Shi W, Smyth GK. limma powers differential expression analyses for RNA-sequencing and microarray studies. Nucleic Acids Res 2015, 43, (7), e47-e47.

17. Wang NN, Dong J, Zhang L, Ouyang D, Cheng Y, Chen AF, et al. HAMdb: a database of human

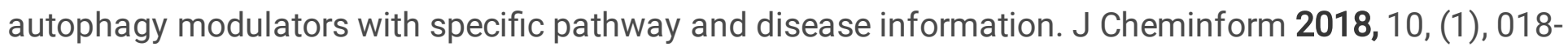
0289.

18. Wang P, Wang Y, Hang B, Zou X, Mao JH. A novel gene expression-based prognostic scoring system to predict survival in gastric cancer. Oncotarget 2016, 7, (34), 55343.

19. Wilkerson MD, Hayes DN.ConsensusClusterPlus: a class discovery tool with confidence assessments and item tracking. Bioinformatics 2010, 26, (12), 1572-3.

20. Linderman GC, Steinerberger S. Clustering with t-SNE, provably. SIAM J Math Data Sci 2019, 1, (2), 313-332.

21. Goel MK, Khanna P, Kishore J. Understanding survival analysis: Kaplan-Meier estimate. Int J Ayurveda Res 2010, 1, (4), 274-278.

22. Dennis GJr, Sherman BT, Hosack DA, Yang J, Gao W, Lane HC, et al. DAVID: Database for Annotation, Visualization, and Integrated Discovery. Genome Biol 2003, 4, (5), P3.

23. Yoshihara K, Shahmoradgoli M, Martínez E, Vegesna R, Kim H, Torres-Garcia W, et al. Inferring tumour purity and stromal and immune cell admixture from expression data. Nat Commun 2013, 4, 2612.

24. Knowles MA, Hurst CD. Molecular biology of bladder cancer: new insights into pathogenesis and clinical diversity. Nat Rev Cancer 2015, 15, (1), 25-41.

25. Richters A, Aben KKH, Kiemeney L. The global burden of urinary bladder cancer: an update. World $\mathrm{J}$ Urol 2020, 38, (8), 1895-1904.

26. Sun Y. Tumor microenvironment and cancer therapy resistance. Cancer Lett 2016, 380, (1), 205-15. 
27. Wang F, Tang J, Li P, Si S, Yu H, Yang X, et al. Chloroquine Enhances the Radiosensitivity of Bladder Cancer Cells by Inhibiting Autophagy and Activating Apoptosis. Cell Physiol Biochem 2018, 45, (1), 54-66.

28. Hu B, Wang Z, Zeng H, Qi Y, Chen Y, Wang T, et al. Blockade of DC-SIGN(+) Tumor-Associated Macrophages Reactivates Antitumor Immunity and Improves Immunotherapy in Muscle-Invasive Bladder Cancer. Cancer Res 2020, 80, (8), 1707-1719.

29. Antonelli AC, Binyamin A, Hohl TM, Glickman MS, Redelman-Sidi G.Bacterial immunotherapy for cancer induces CD4-dependent tumor-specific immunity through tumor-intrinsic interferon-y signaling. $P$ Natl Acad Sci USA 2020, 117, (31), 18627-18637.

30. Coleman Ol, Lobner EM, Bierwirth S, Sorbie A, Waldschmitt N, Rath E, et al. Activated ATF6 Induces Intestinal Dysbiosis and Innate Immune Response to Promote Colorectal Tumorigenesis.

Gastroenterology 2018, 155, (5), 1539-1552.

31. Zhang HH, Li C, Ren JW, Liu L, Du XH, Gao J, et al. OTUB1 facilitates bladder cancer progression by stabilizing ATF6 in response to endoplasmic reticulum stress. Cancer Sci 2021, 112, (6), 2199-2209.

32. Meng J, Liu K, Shao Y, Feng X, Ji Z, Chang B, et al. ID1 confers cancer cell chemoresistance through STAT3/ATF6-mediated induction of autophagy. Cell Death Dis 2020, 11, (2), 020-2327.

33. Sharif T, Ahn DG, Liu RZ, Pringle E, Martell E, Dai C,et al. The NAD(+) salvage pathway modulates cancer cell viability via p73. Cell Death Differ 2016, 23, (4), 669-80.

34. Zhang K, Zhou B, Zhang P, Zhang Z, Chen P, Pu Y, et al. Genetic variants in NAMPT predict bladder cancer risk and prognosis in individuals from southwest Chinese Han group. Tumour Biol 2014, 35, (5), 4031-40.

35. Chen CH, Changou CA, Hsieh TH, Lee Y C, Chu CY, Hsu KC, et al. Dual Inhibition of PIK3C3 and FGFR as a New Therapeutic Approach to Treat Bladder Cancer. Clin Cancer Res 2018, 24, (5), 1176-1189.

36. Chen $\mathrm{CH}$, Hsieh TH, Lin YC, Liu YR, Liou JP, Yen Y. Targeting Autophagy by MPTOL145, a Highly Potent PIK3C3 Inhibitor, Provides Synergistic Interaction to Targeted or Chemotherapeutic Agents in Cancer Cells. Cancers 2019, 11, (9), 1345-1358.

\section{Tables}

Table 1. The 35 autophagy-related genes associated with prognosis according to the univariate Cox regression analysis. 


\begin{tabular}{|c|c|c|}
\hline Genes & Median absolute deviation (MAD) & $\mathrm{p}$ value \\
\hline APOL 1 & 128.930 & $1.48 \mathrm{E}-03$ \\
\hline CTSB & 91.449 & $3.18 \mathrm{E}-02$ \\
\hline$P 4 H B$ & 67.674 & $1.90 \mathrm{E}-05$ \\
\hline ITGB1 & 23.824 & $3.21 \mathrm{E}-02$ \\
\hline EIF4G1 & 18.062 & 7.35E-03 \\
\hline$P R K C D$ & 13.842 & $2.04 \mathrm{E}-02$ \\
\hline VEGFA & 13.232 & $1.72 \mathrm{E}-02$ \\
\hline LAMP2 & 12.719 & $3.15 \mathrm{E}-02$ \\
\hline CAPN2 & 12.488 & $2.02 \mathrm{E}-03$ \\
\hline NAMPT & 10.530 & 1.30E-03 \\
\hline$B A G 1$ & 8.101 & 3.39E-02 \\
\hline SPHK1 & 7.940 & $4.68 \mathrm{E}-02$ \\
\hline ATIC & 7.714 & $2.62 \mathrm{E}-02$ \\
\hline MAPK3 & 6.494 & $2.26 \mathrm{E}-03$ \\
\hline CASP1 & 5.773 & $2.72 \mathrm{E}-02$ \\
\hline ATF6 & 3.778 & $1.24 \mathrm{E}-04$ \\
\hline WDR45 & 3.232 & $2.96 \mathrm{E}-03$ \\
\hline MAPK1 & 3.092 & $4.61 \mathrm{E}-02$ \\
\hline WIPI1 & 2.841 & 4.31E-03 \\
\hline ATG9A & 2.712 & $2.25 \mathrm{E}-02$ \\
\hline$A T G 4 B$ & 2.701 & $9.25 \mathrm{E}-03$ \\
\hline STK11 & 2.519 & $3.74 \mathrm{E}-03$ \\
\hline NPC1 & 2.330 & $6.98 \mathrm{E}-03$ \\
\hline$I K B K B$ & 2.188 & 4.29E-03 \\
\hline MTOR & 1.840 & $2.66 \mathrm{E}-02$ \\
\hline$R A B 24$ & 1.578 & $2.84 \mathrm{E}-03$ \\
\hline CASP8 & 1.569 & $3.00 \mathrm{E}-02$ \\
\hline RPTOR & 1.248 & $1.48 \mathrm{E}-03$ \\
\hline
\end{tabular}

Page 12/20 


\begin{tabular}{|lll|} 
NRG1 & 1.189 & $1.26 \mathrm{E}-02$ \\
ULK2 & 1.077 & $4.53 \mathrm{E}-02$ \\
CAPN10 & 0.920 & $1.26 \mathrm{E}-02$ \\
\hline IFNG & 0.790 & $4.50 \mathrm{E}-02$ \\
\hline ARSB & 0.788 & $2.96 \mathrm{E}-03$ \\
\hline PIK3C3 & 0.641 & $1.21 \mathrm{E}-02$ \\
\hline DIRAS3 & 0.634 & $1.32 \mathrm{E}-02$ \\
\hline
\end{tabular}

Table 2. The 7 genes identified as independent prognostic signatures by multivariable Cox regression.

\begin{tabular}{|lll|}
\hline Genes & coef & p value \\
\hline ATF6 & 0.027 & $6.82 \mathrm{E}-03$ \\
\hline CAPN2 & 0.014 & $3.85 \mathrm{E}-03$ \\
\hline NAMPT & 0.010 & $3.62 \mathrm{E}-03$ \\
\hline NPC1 & 0.051 & $1.19 \mathrm{E}-03$ \\
\hline P4HB & 0.002 & $2.14 \mathrm{E}-03$ \\
\hline PIK3C3 & 0.146 & $6.49 \mathrm{E}-04$ \\
\hline RPTOR & 0.161 & $2.57 \mathrm{E}-04$ \\
\hline
\end{tabular}

Figures 


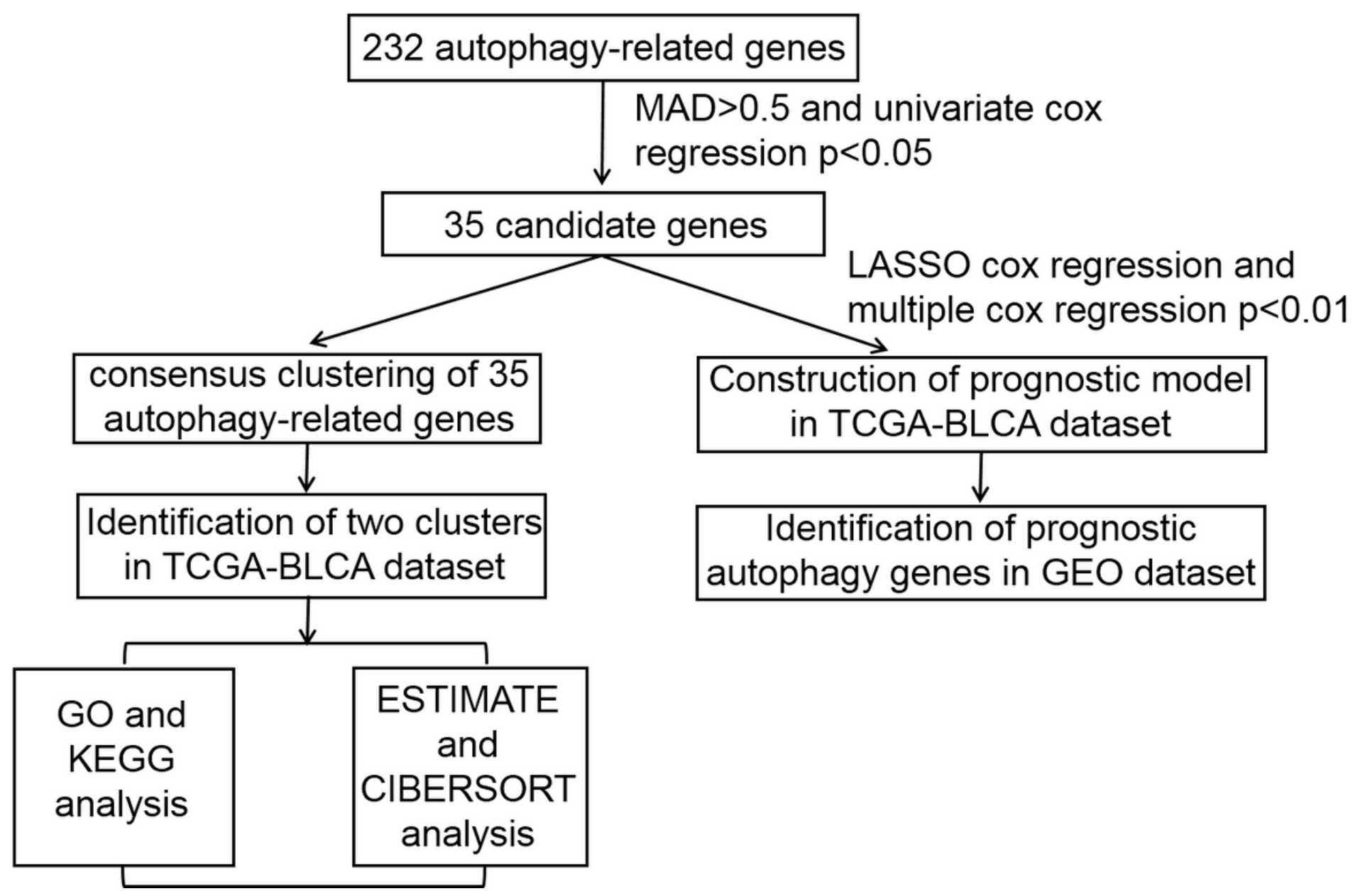

Figure 1

Flow chart of the study. 
A

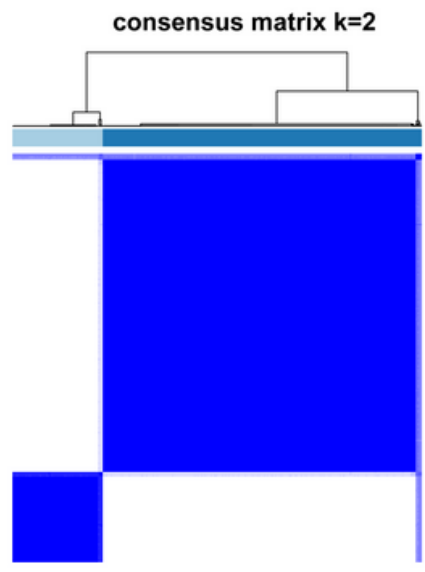

D
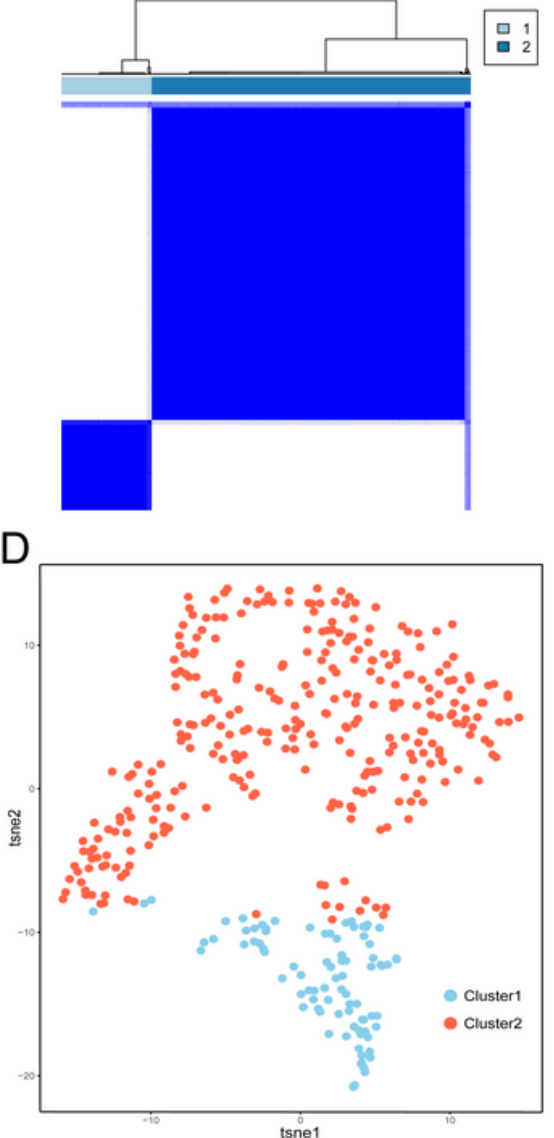

B

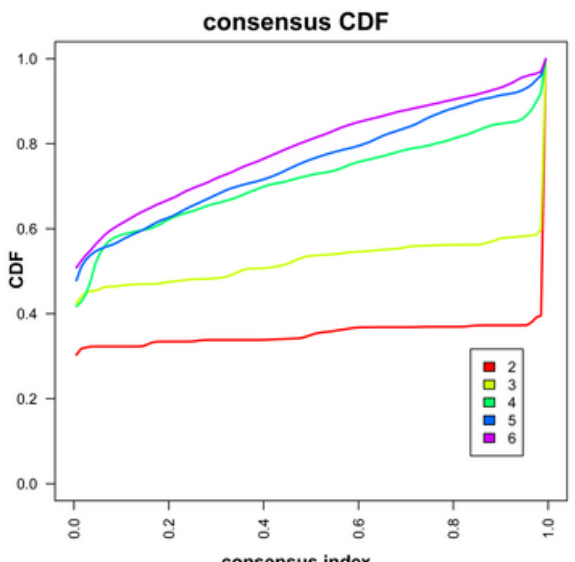

$\mathrm{E}$

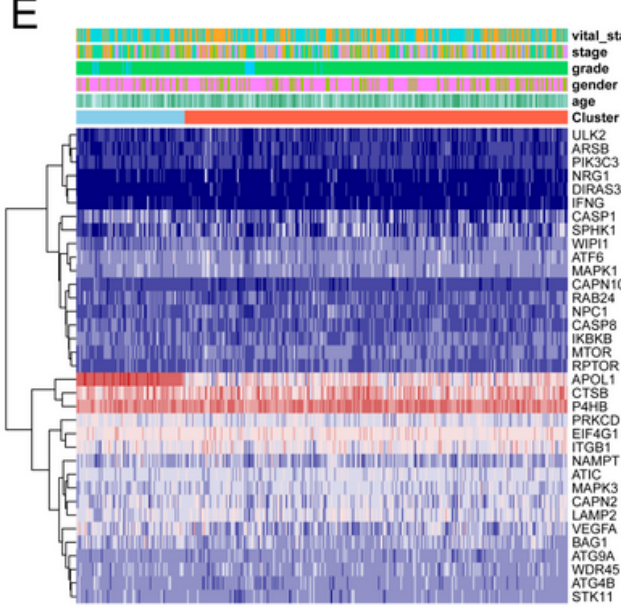

C

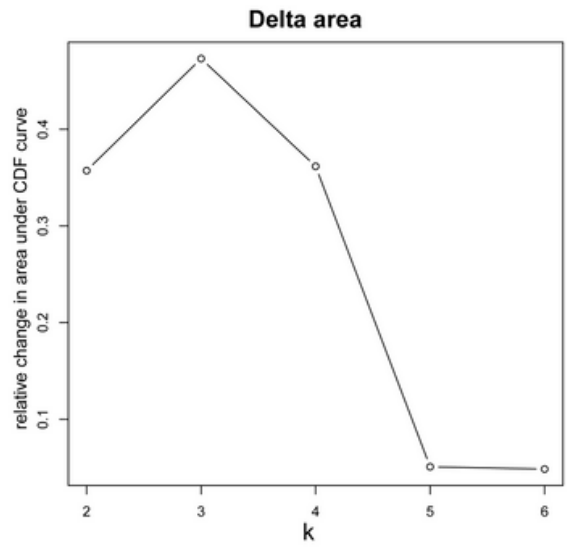

$\mathrm{F}$

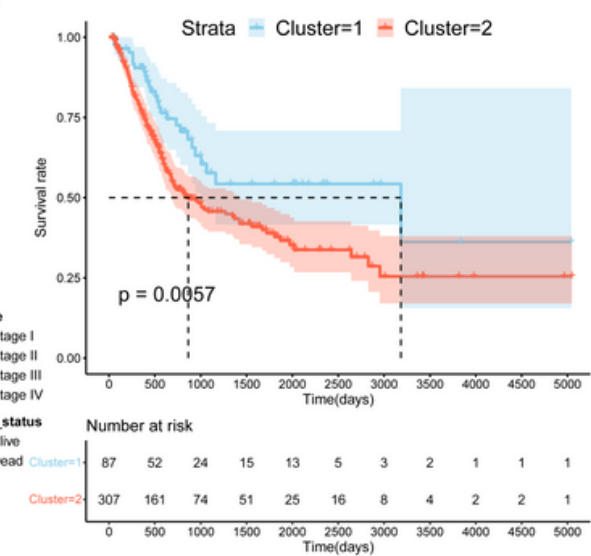

Figure 2

Consensus clustering of autophagy-related genes. (A) Consensus clustering matrix for $k=2$. (B) Consensus clustering cumulative distribution function (CDF) for $k=2$ to 6 . (C) The relative change in area under CDF curve for $\mathrm{k}=2$ to 6 . (D) t-distributed stochastic neighbour embedding (t-SNE) algorithm supported the stratification into two BLCA subclasses. (E) Heatmap of two clusters defined by the expressions of autophagy-related genes. (F) Survival analysis of patients in cluster 1 and cluster 2 in TCGA-BLCA cohort. 
GO:0016032 viral process
GO:0043123 positive regulation of I-kappaB kinase/NF-kappaB signaling GO:0060333 interferon-gamma-mediated signaling pathway

GO:0009615 response to virus

GO:0060337 type I interferon signaling pathway

GO:0002479 antigen processing and presentation of exogenous peptide antigen via MHC class I

GO:0034097 response to cytokine

GO:0002474 antigen processing and presentation of peptide antigen via MHC class I

GO:0002053 positive regulation of mesenchymal cell proliferation

GO:0042113 B cell activation

GO:0033077 T cell differentiation in thymus

GO:0019885 antigen processing and presentation of endogenous peptide antigen via MHC class I

GO:2000778 positive regulation of interleukin- 6 secretion

GO:0032731 positive regulation of interleukin-1 beta production -

GO:0012501 programmed cell death

GO:0001783 B cell apoptotic process

GO:0032611 interleukin-1 beta production

GO:0002829 negative regulation of type 2 immune response

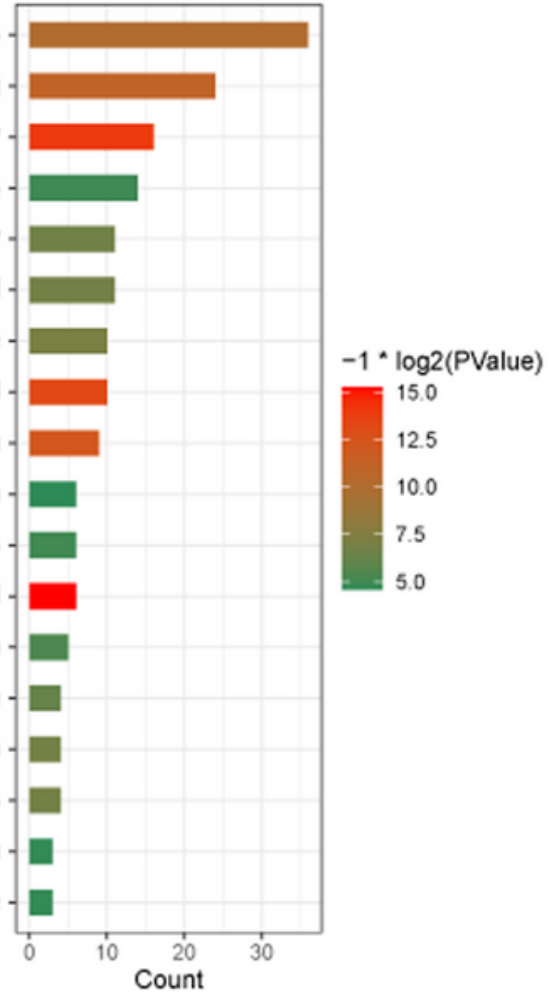

$\mathrm{B}$

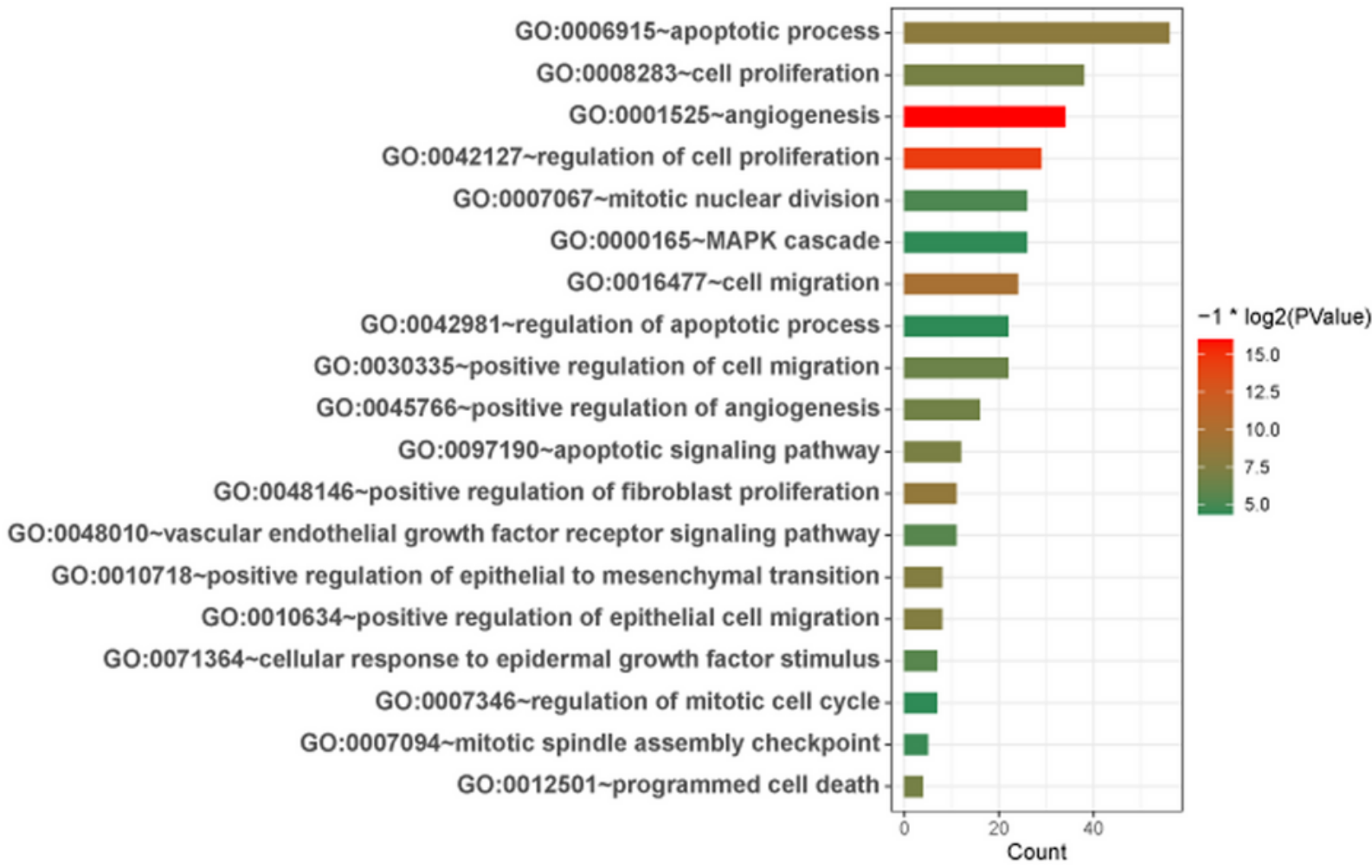

\section{Figure 3}

Gene ontology (GO) functional enrichment for differentially expressed genes (DEGs). (A) GO terms related to immune response. (B) GO terms related to cancer 


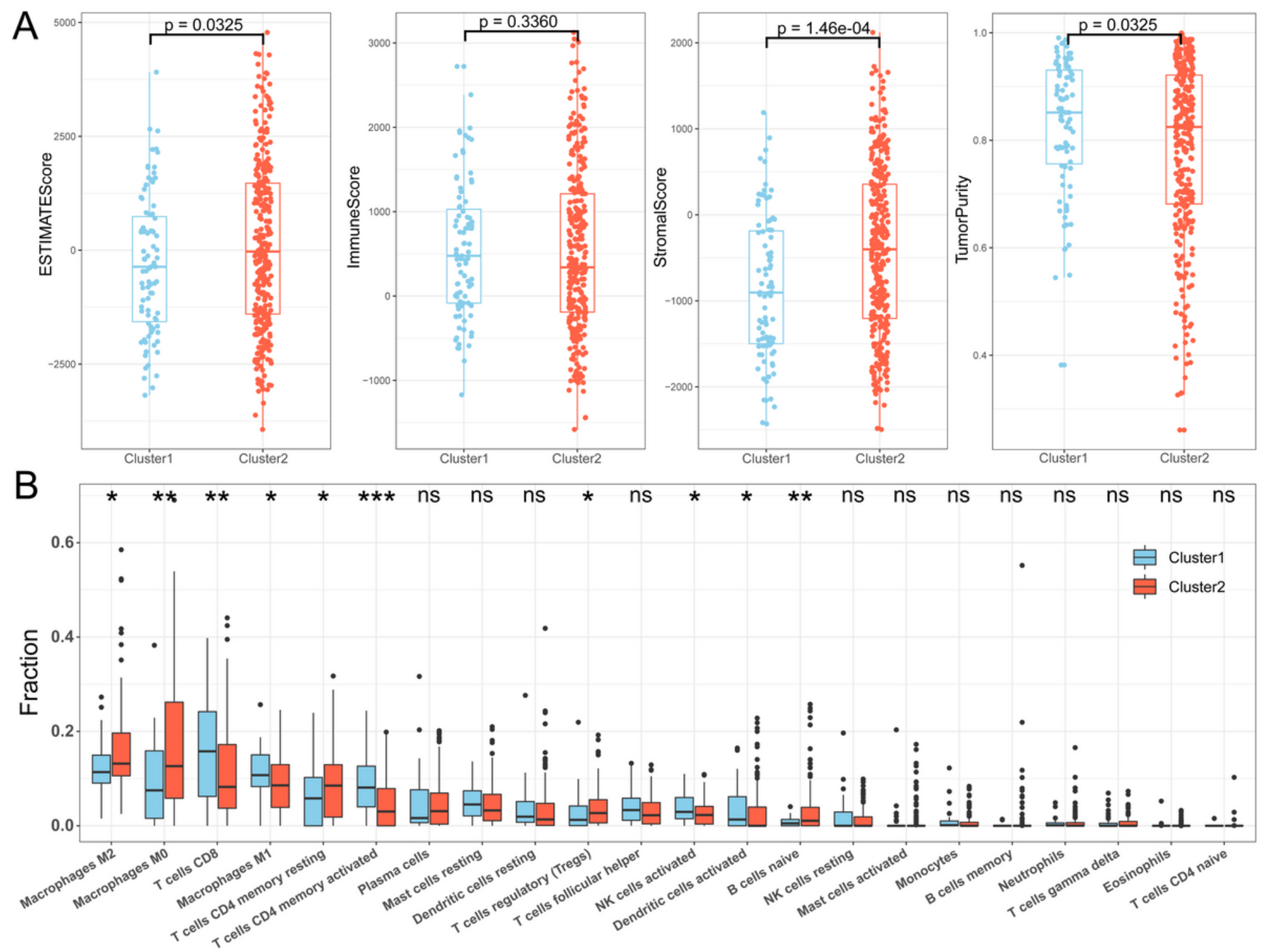

\section{Figure 4}

Immune characteristics of the two clusters. (A) Differences in the values of estimate score, immune score, stromal score, and tumor purity between the two clusters. (B) Differences in the infiltrations of the 22 immune cells between the two clusters ( ${ }^{\star \star *} p<0.001 ;{ }^{* \star} p<0.01 ;{ }^{*} p<0.05$; ns: not significant). 

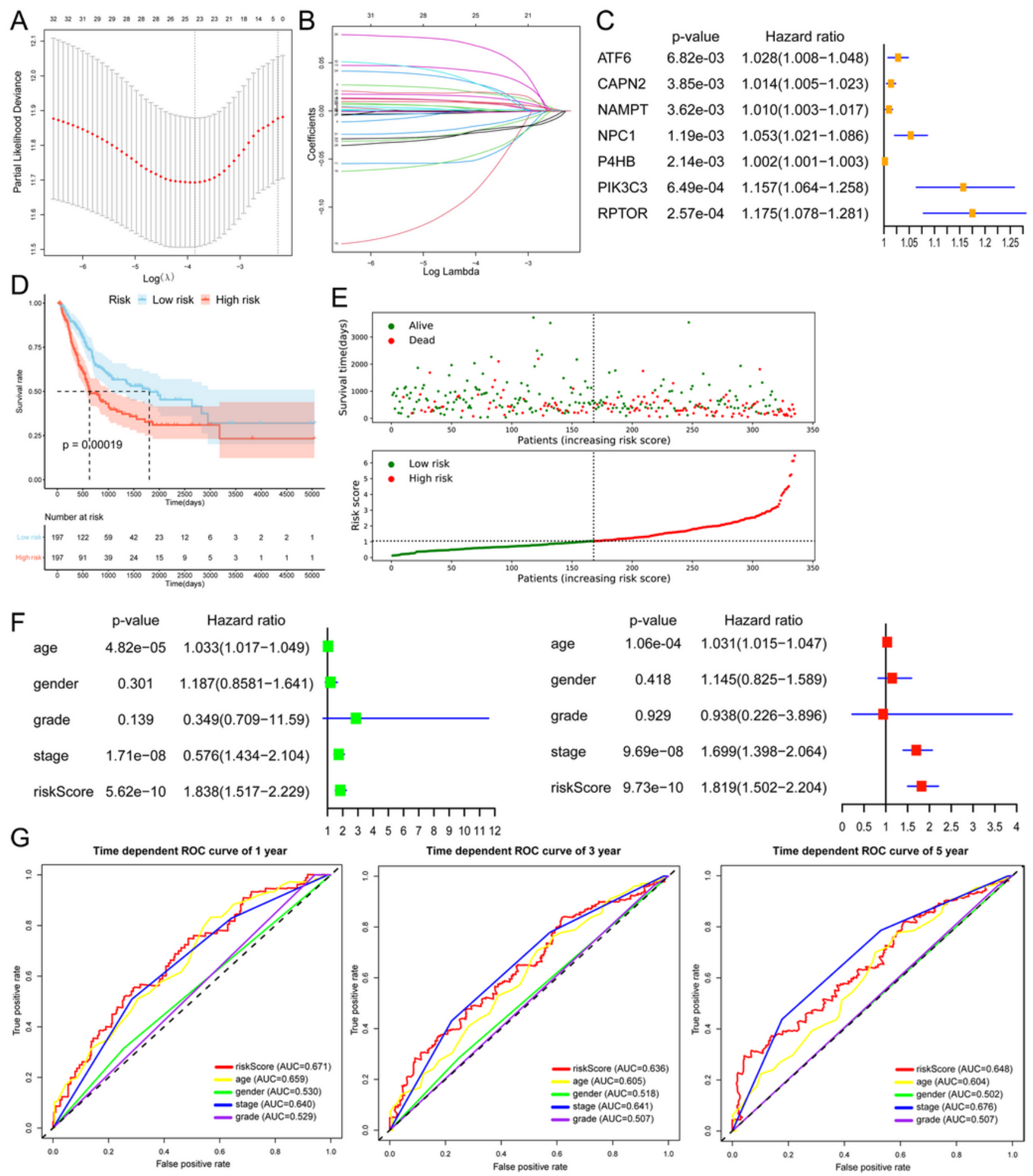

\section{Figure 5}

Prognostic model based on autophagy-related genes. (A) Lasso Cox regression analysis of autophagyrelated genes. (B) Lasso coefficient values were calculated at the best log (lambda) value. (C): Forest plot of multivariate cox regression analysis for 7 autophagy-related genes. The Hazard Ratio (HR) value and its $95 \%$ confidence interval, as well as associated p-value, were showed. (D): Kaplan-Meier curves showed that the high-risk group had worse survival probability than the low-risk group. (E): Scatter plot showed 
the correlation between the survival status and risk score; Risk score distribution plot showed the distribution of high-risk and low-risk patients; (F): Validation of the independence of the autophagyrelated genes in OS through the univariate cox regression analysis and multivariate cox regression analysis. (G): The ROC curves of risk score and clinical index in 1, 3, 5 years survival probability.
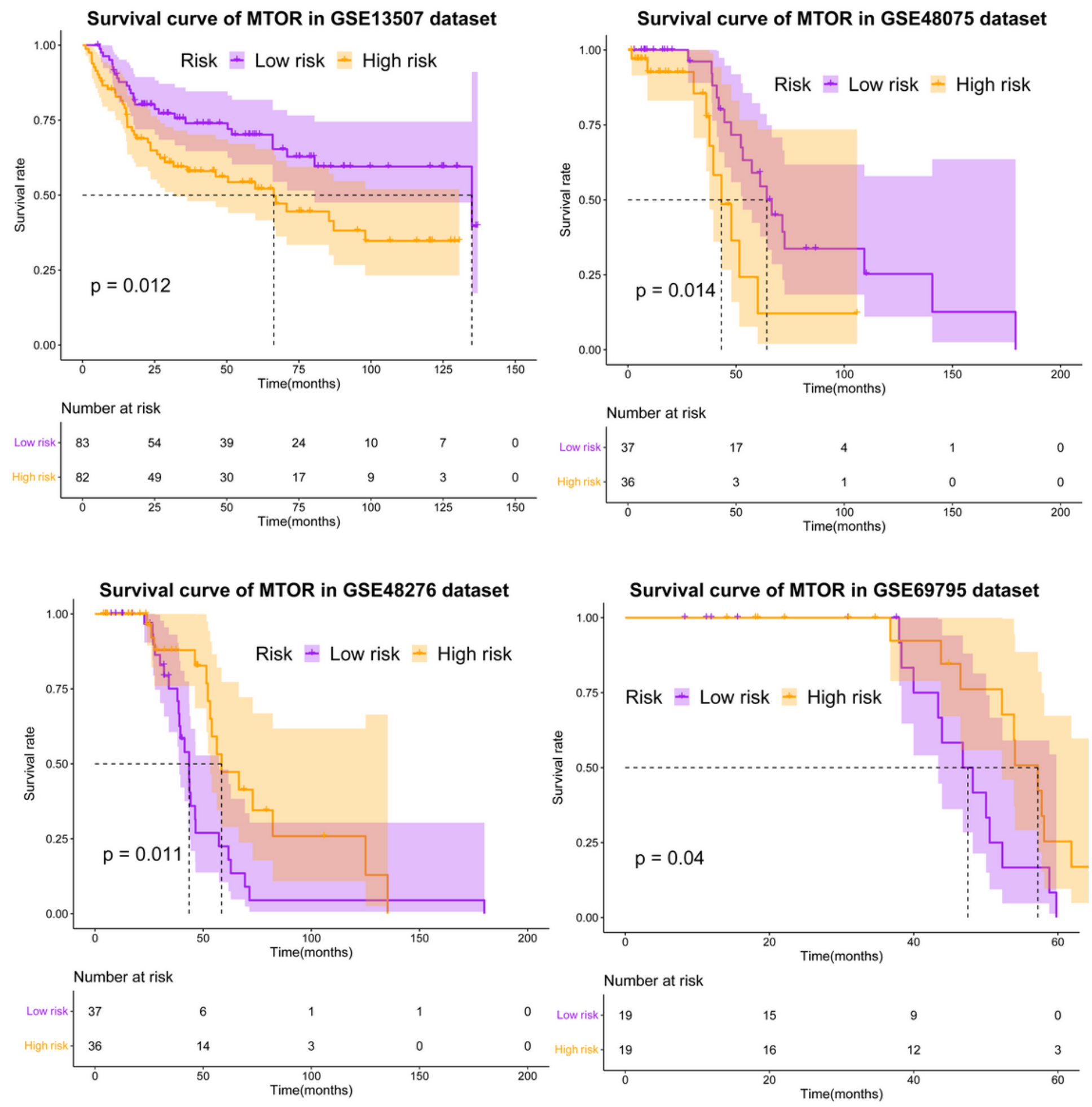

Figure 6 
Survival curve of MTOR in GEO datasets GSE13507, GSE48075, GSE48276 and GSE69795.

\section{Supplementary Files}

This is a list of supplementary files associated with this preprint. Click to download.

- Supplementarytable1.xlsx

- Supplementarytable2.xlsx

- Supplementarytable3.xlsx

- Supplementarytable4.xlsx

- Supplementarytable5.xlsx

- Supplementarytable6.xlsx

- Figures1.tif

- Figures2.tif 